\title{
Cistectomía Radical Laparoscópica
}

\author{
Castillo $\mathrm{O}^{1,2}$, Cabello Benavente $\mathrm{R}^{3}$, Briones Mardones $\mathrm{G}^{4}$, Hernández Fernández $\mathrm{C}^{3}$. \\ ${ }^{1}$ Unidad de Endourología y Laparoscopia Urológica, Clínica Santa María, Santiago, Chile. \\ ${ }^{2}$ Departamento de Urología, Facultad de Medicina, Universidad de Chile, Santiago, Chile. \\ ${ }^{3}$ Servicio de Urología, Hospital Gregorio Marañón, Madrid. \\ ${ }^{4}$ Servicio de Urología, Hospital Son Llatzer, Mallorca.
}

Actas Urol Esp 2006; 30 (5): 531-540

\section{RESUMEN}

CISTECTOMÍA RADICAL LAPAROSCÓPICA

La cistectomía radical laparoscópica está siendo introducida en la mayoría de los grupos que tienen demostrada experiencia en cirugía laparoscópica y sobre todo en aquellos que han superado la curva de aprendizaje en prostatectomía radical laparoscópica.

Se considera aún una técnica de alta complejidad, que no está aún al alcance de la mayoría de los Servicios de Urología.

Revisamos en este trabajo la técnica quirúrgica así como la literatura al respecto, haciendo hincapié en sus indicaciones, ventajas y morbilidad más habitual.

Palabras clave: Laparoscopia. Cistectomía radical.

\section{ABSTRACT \\ RADICAL LAPAROSCOPIC CYSTECTOMY}

Radical laparoscopic cystectomy is being adopted by most groups with proven experience in laparoscopic surgery, especially by those who have already completed the learning curves in radical laparoscopic prostatectomy.

It is still considered a highly complex technique, which has not yet been mastered in many Urology Units.

In this article, we review the surgical technique and available literature on this approach, with special emphasis on its indications, advantages and most frequent morbidity.

Keywords: Laparoscopy. Radical cystectomy.

$\mathrm{L}$ a laparoscopia inicialmente fue una herramienta meramente diagnóstica o ablativa sobre los órganos intra-abdominales. Hoy en día es utilizada por casi todas las ramas de la cirugía quirúrgica en procedimientos de alta complejidad técnica. Todo ello gracias a la progresiva adquisición de destreza y confianza por parte de los cirujanos y a la mejora en la tecnología disponible $^{1}$. El desarrollo de la laparoscopia en urología se ha retrasado en relación con otras especialidades, hecho que tal vez esté relacionado con el difícil acceso a sus órganos diana retroperitoneales y con la necesidad de técnicas complejas de reconstrucción en la cirugía vesical y prostática ${ }^{2}$.

La cistoprostatectomía radical abierta (CPRA) es el "gold standard" para el tratamiento quirúrgico del cáncer vesical infiltrante no metastático y superficial de alto riesgo ${ }^{3}$. El desarrollo laparoscópico en urología ha permitido proponer a la cistoprostatectomía radical laparoscópica (CPRL) como una alternativa a la cirugía abierta. Desde que las primeras comunicaciones fueran publicadas en el primer lustro de los 
años 1990 Sánchez de Badajoz ${ }^{4}$, Puppo ${ }^{5}$ y tras una década de evolución, la CPRL continúa siendo una técnica pionera, realizada exclusivamente en centros con amplia experiencia en laparoscopia $^{6,7}$.

Demostrar la viabilidad de la CPRL no significa que esta deba reemplazar la alternativa tradicional. La mayoría de la experiencia publicada se centra en la descripción de la técnica, faltan datos sobre el seguimiento y supervivencia a largo plazo antes de poder obtener conclusiones válidas. Los primeros casos reportados demuestran que es una técnica viable y que probablemente será capaz de igualar o incluso superar los resultados de la CPRA ${ }^{6}$.

\section{TÉCNICA GUIRÚRGICA}

\section{Cistectomía}

La técnica que describimos a continuación corresponde al desarrollo de un procedimiento totalmente autodidacta, desarrollado luego de haber perfeccionado la técnica de prostatectomía radical laparoscópica.

En la preparación del paciente, el día anterior a la cirugía se le da una dieta líquida y se le indica la ingestión de un frasco de Fosfosoda oral a las 8 AM y $17 \mathrm{PM}$. El paciente es ingresado la noche anterior a la cirugía, donde se le coloca un Enema Fosfosoda evacuante del recto y se inicia terapia con Heparina de bajo peso molecular.

Bajo anestesia general el paciente se coloca en posición de litotomía modificada (Fig. 1), la cual permite tener acceso al recto en caso de lesión de éste, y al mismo tiempo permite presionar el periné para facilitar una eventual anastomosis a la uretra. En la inducción anestésica se coloca una sonda naso-gástrica y se usa una primera dosis de una Cefalosporina de $1^{\underline{a}}$ generación y Metronidazol, la cuales son repetidas a las 3 horas de la cirugía si es que el procedimiento se prolonga. La sonda vesical se coloca en el campo quirúrgico para su futura manipulación.

Se utiliza apoyo en los hombros para usar luego una posición de Trendelenburg forzada y se inicia la cirugía realizando un neumoperitoneo con punción con aguja de Veress hasta $15 \mathrm{mmHg}$ en posición supraumbilical. Los puertos de trabajo se colocan de la siguiente manera: un trocar de 10-12 mm supraumbilical para la óptica de $0^{\circ}$,

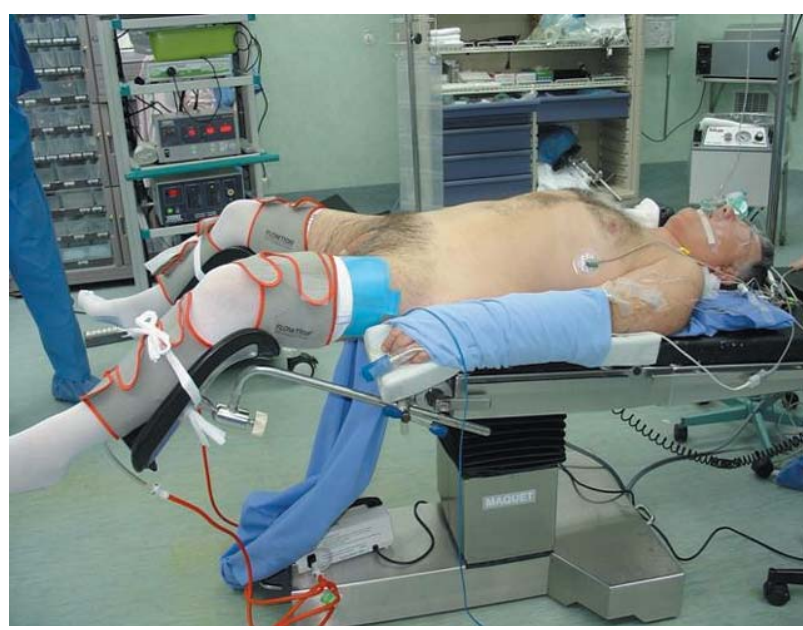

FIGURA 1

2 puertos de 10-12 $\mathrm{mm}$ en ambas fosas ilíacas, laterales a los vasos epigástricos y a mitad de camino entre el ombligo y el pubis, y 2 trocares de $5 \mathrm{~mm}$ paraumbilicales, conformando una figura en $\mathrm{W}$ (Fig. 2).

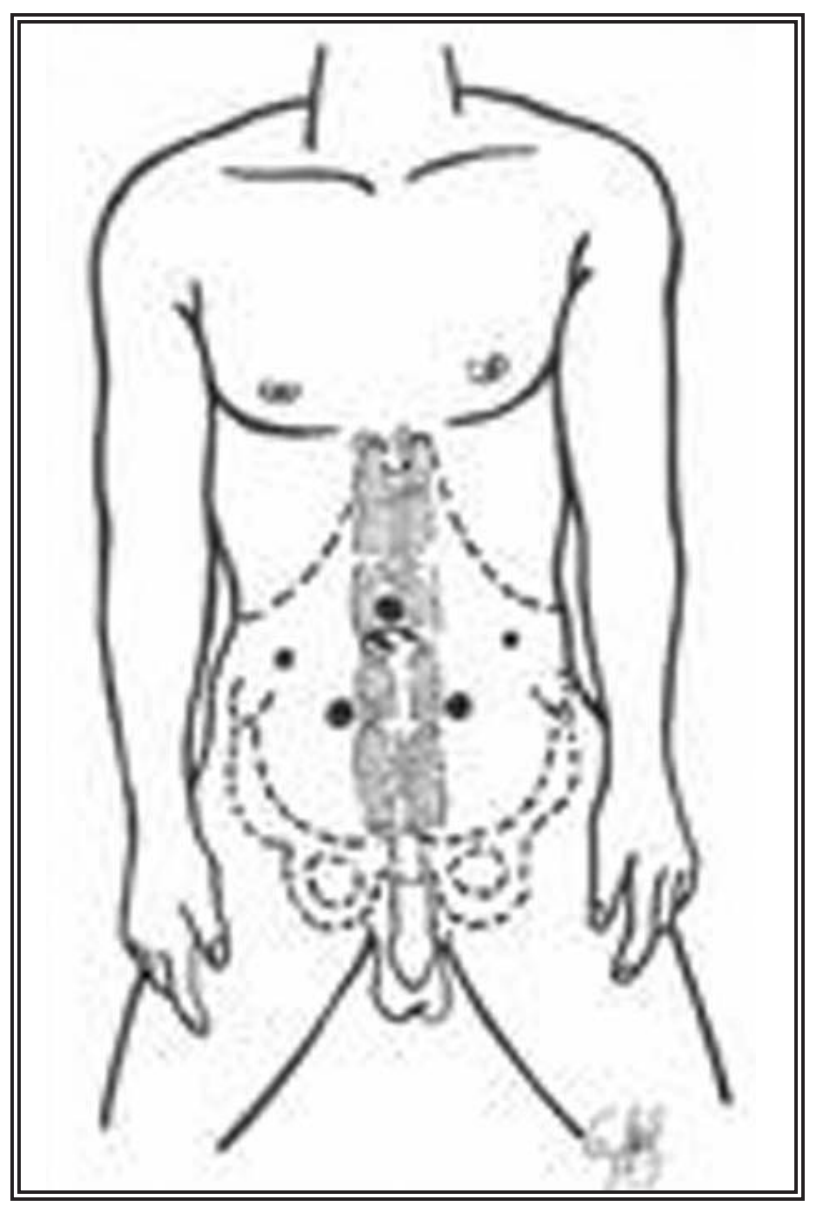

FIGURA 2 
La cirugía se inicia realizando una incisión del peritoneo en forma de U, la cual incluye el fondo de saco recto-vesical en la base y una línea que va entre el ligamento umbilical lateral y el cordón espermático (Fig. 3), seccionado los conductos deferentes. Para el procedimiento utilizamos una pinza bipolar en la mano izquierda y el bisturí armónico (Ethicon Endosurgery) en la mano derecha. Se identifica el uréter y se secciona entre Hem-O-Lok, enviando un segmento para biopsia por congelación. Luego se procede a realizar una linfadenectomía amplia que comprende el nervio genitofemoral lateral, la vejiga medial, el pubis distal y la bifurcación ilíaca proximal (Fig. 4). Este tiempo toma aproximadamente $60 \mathrm{minu}-$ tos para la linfadenectomía bilateral. El tejido se coloca en una bolsa y se fija a uno de los puertos de $5 \mathrm{~mm}$ laterales para ser retirado junto con la pieza de cistectomía.

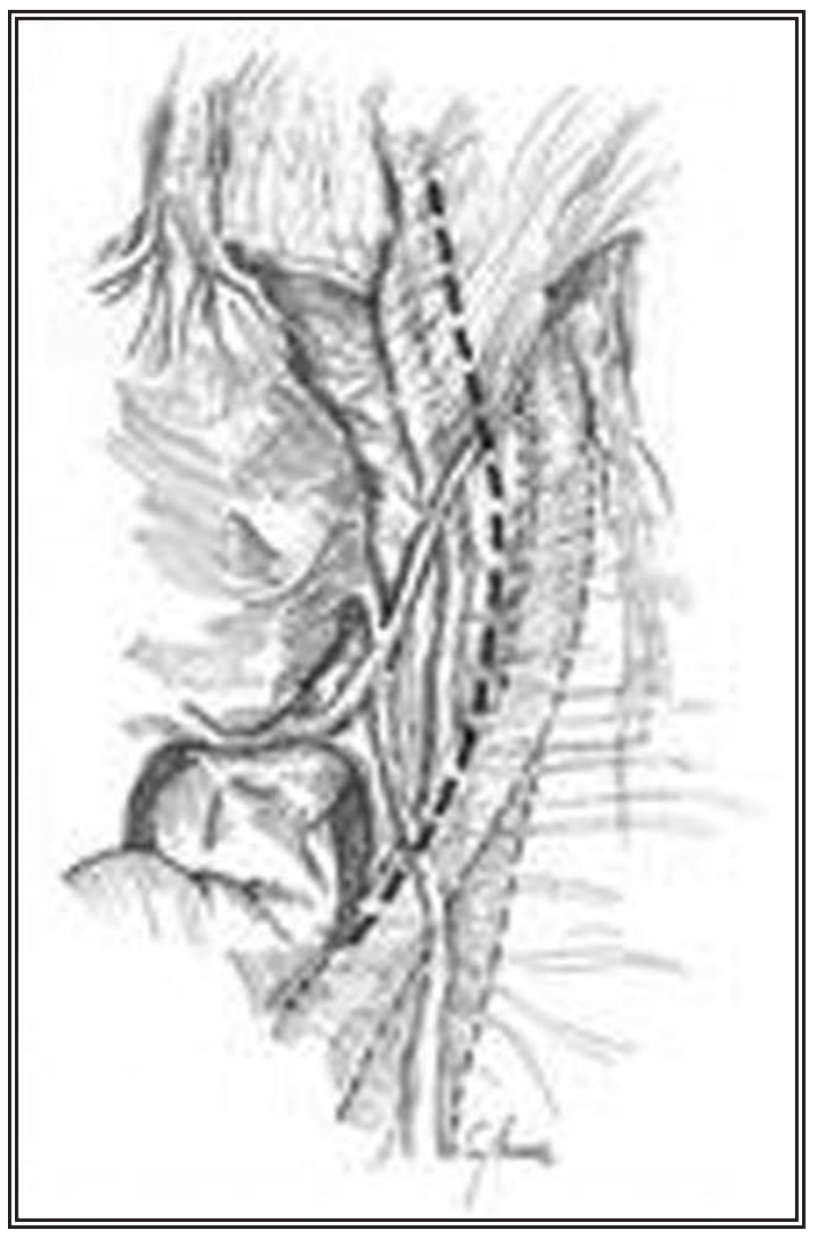

FIGURA 3

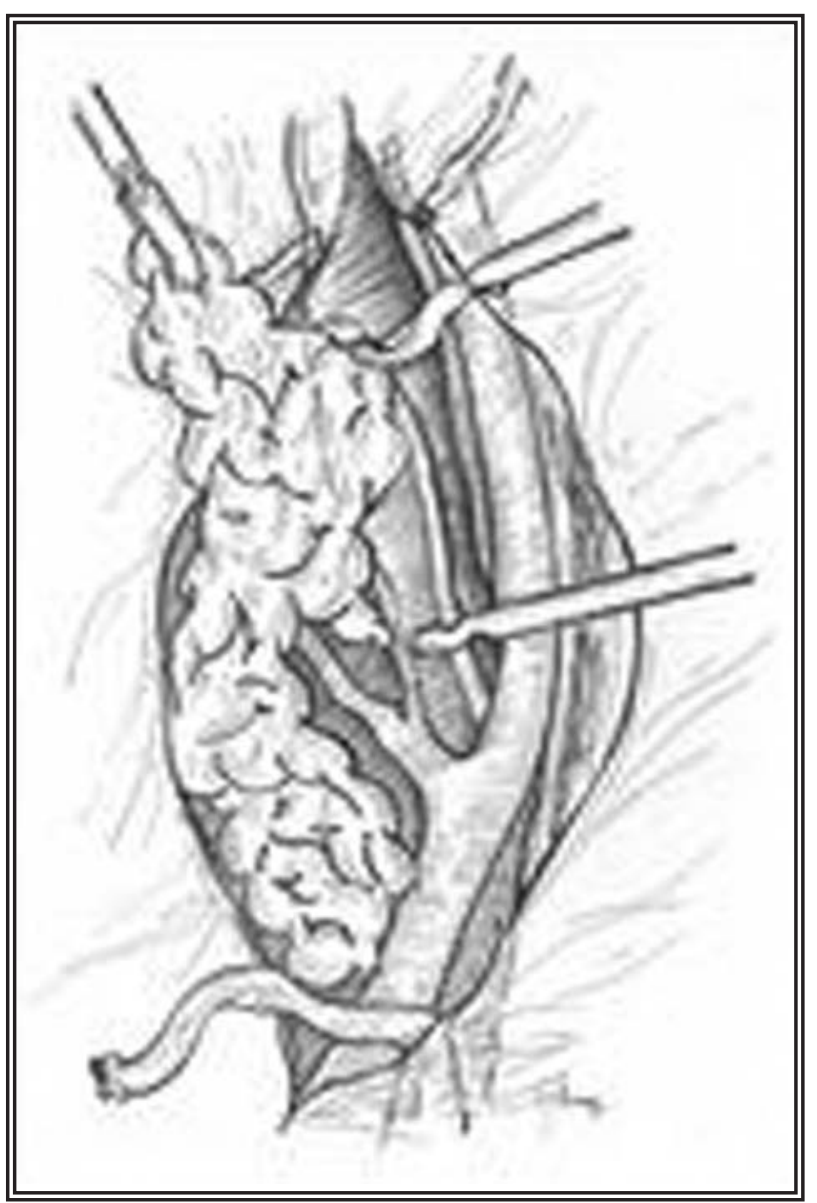

FIGURA 4

Preferimos hacer la linfadenectomía primero, pues luego es muy fácil identificar los pedículos vesicales para proceder a la cistectomía.

El tiempo de la cistectomía es como sigue. Al momento de haber seccionado el peritoneo del fondo de saco identificamos las vesículas seminales pero no las disecamos, para que salgan en bloque con el espécimen. Seccionamos la hoja posterior de Denovilliers y avanzamos controlando los pedículos vesicales superior y medio, para lo cual inicialmente utilizamos Endo-Gia (Ethicon Endosurgery), luego Ligasure (ValleyLab), y últimamente la nueva pinza del bisturí armónico (Ethicon Endosurgery). Una vez controlados los pedículos vesicales, procedemos a seccionar el peritoneo parietal anterior para bajar la vejiga y entrar al espacio de Retzius. Al tener el puerto de la cámara en posición supraumbilical podemos seccionar el uraco, sin riesgo de lesionar la vejiga. 
Abrimos con tijera fría la fascia endopélvica a ambos lados, seccionamos los ligamentos puboprostáticos y pasamos un punto en 8 de Vycril 0 con aguja CT-1 al complejo venoso dorsal (paso idéntico al de la prostatectomía radical). En este momento traccionamos la sonda vesical por fuera y la anudamos, para luego poder cortarla por dentro sin perder el balón.

Seccionamos el complejo dorsal y luego la cara anterior de la uretra, identificando la sonda. Traccionamos la sonda hacia el interior, hasta identificar las ligaduras hechas en forma externa, y seccionamos la sonda. El ayudante toma la sonda con una pinza fuerte y la tracciona hacia cefálico, con lo cual exponemos la cara posterior de la uretra, que se secciona con tijera, y podemos continuar con la disección en forma retrógrada hasta seccionar los pedículos prostáticos, liberando la pieza. Esta es colocada en una bolsa de polietileno para ser extraída, evitando la eventual contaminación con orina del campo quirúrgico y el riesgo de implante de células tumorales.

Para la extracción de la pieza hemos usado una incisión infraumbilical de $6 \mathrm{~cm}$ (Fig. 5) o una incisión de Pfanestiel.

\section{Derivación urinaria}

En todos los casos hemos realizado la derivación urinaria en forma totalmente extracorpórea. Parece lógico que si es necesario realizar una incisión para extraer la pieza, por esta se pueda realizar todo el trabajo intestinal.

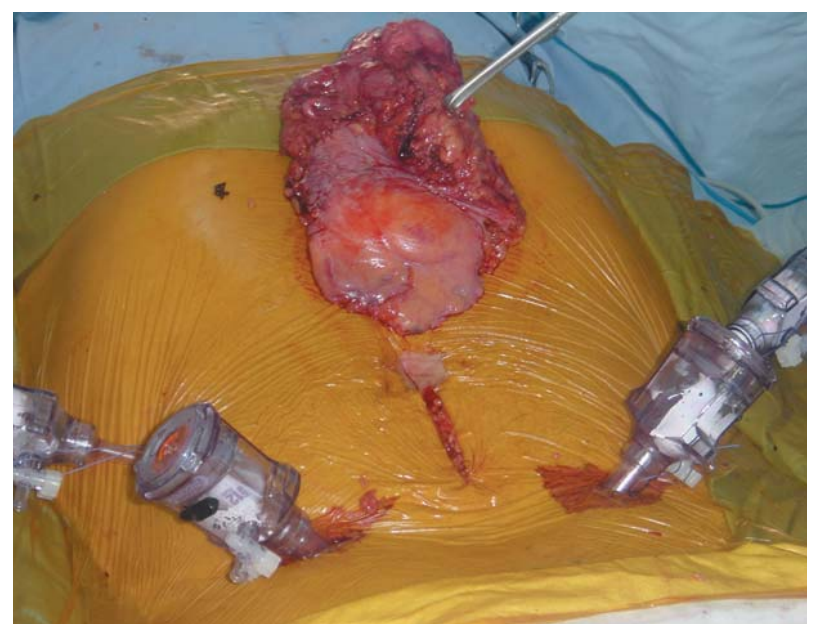

FIGURA 5
En nuestra experiencia sobre 45 cistectomías radicales laparoscópicas, hemos efectuado 3 tipos de derivación urinaria:

Neovejiga ileal ortotópica tipo Studer: El segmento intestinal previamente seleccionado por la vía laparoscópica, midiendo que llegue a la uretra, es exteriorizado por la incisión y se desfuncionaliza un asa de $45 \mathrm{~cm}$ a $25 \mathrm{~cm}$. de la válvula ileo-cecal. El intestino se secciona con un TLC-55 y luego se reconstituye el tránsito con una sutura latero-lateral con los extremos enfrentados con Monocryl 3-0 en un plano total, la cual funciona como una anastomosis término-terminal. Se reconfigura la neovejiga como está descrito y ambos uréteres son anastomosados a la chimenea isoperistática con una amplia espatulación, con Monocryl 5-0, sobre tutores 8 Fr exteriorizados por el asa.

Una vez construido el reservorio (Fig. 6), se reintroduce en la cavidad abdominal y se cierra la incisión para rehacer el neumoperitoneo. $\mathrm{El}$ ayudante toma con una pinza firme el extremo del reservorio y se realiza una anastomosis a la uretra con 6 puntos de Vycril 2-0 con aguja UR-6 (Fig. 7), dejando una sonda Foley Silastic 22 Fr. Los tutores ureterales son exteriorizados por uno de los trocares de 10-12 mm y se deja un drenaje aspirativo en la cavidad pélvica (Fig. 8).

Conducto ileal: Con el paciente de pié, antes de iniciar la cirugía, se marca la posición del estoma, dado que la distensión del neumoperitoneo modifica los puntos de reparo, y el estoma

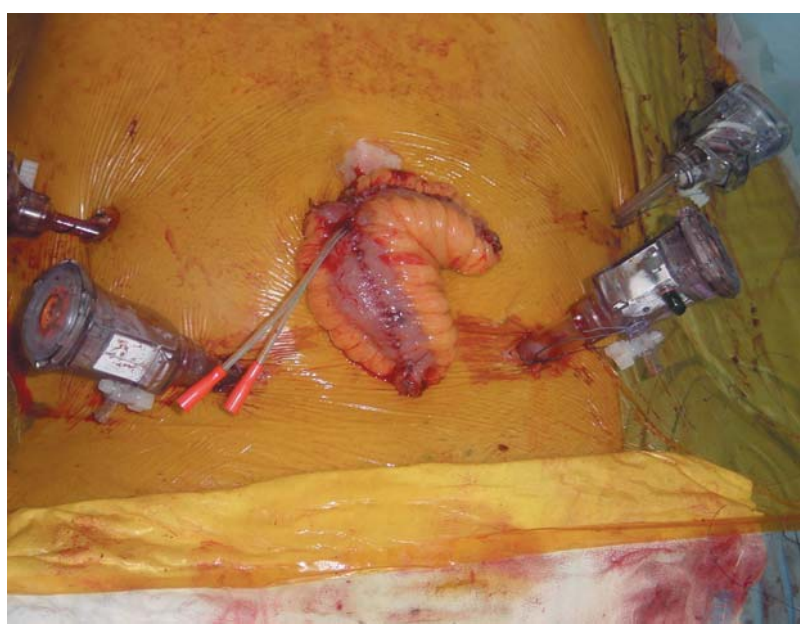

FIGURA 6 


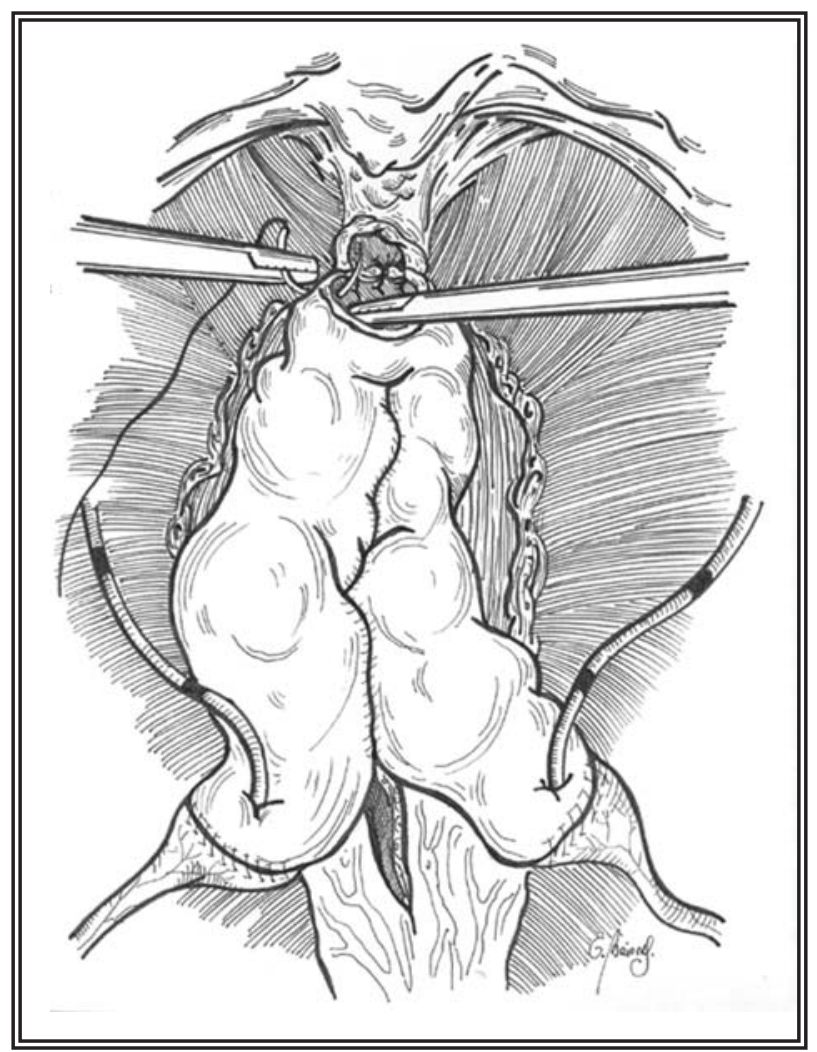

FIGURA 7

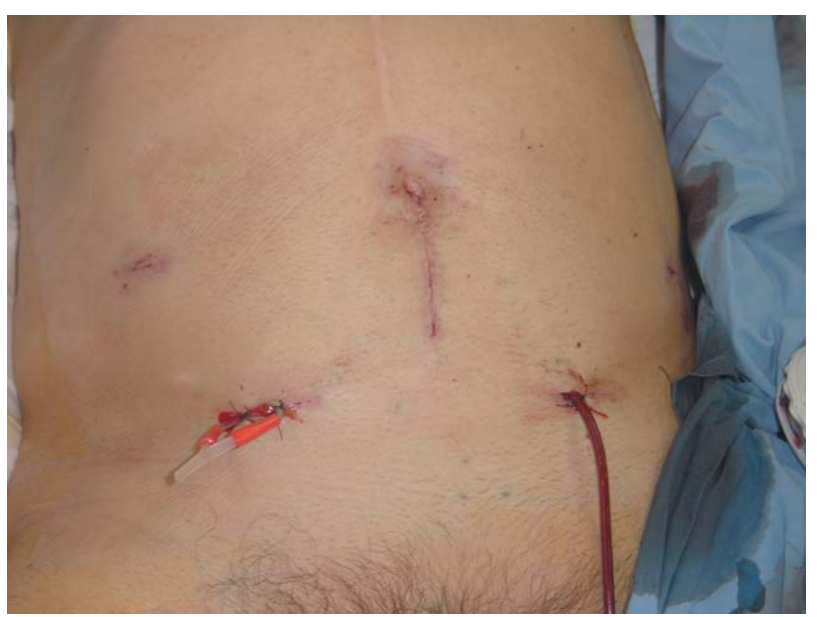

FIGURA 8

puede quedar en posición inadecuada. El trabajo intestinal se realiza de igual forma si se ha necesitado de una incisión para retirar la pieza. En el caso de las mujeres, donde extraemos la pieza por la vagina, se exterioriza el intestino y los uréteres por el sitio del estoma, habiendo realizado la sección en cruz de la aponeurosis. Aunque parezca difícil de imaginar, la distensión abdominal del neumoperitoneo previo provoca tal relajación de la pared abdominal que no hay dificultad para trabajar por el estoma (Fig. 9). El único paso distinto a la neovejiga ortotópica, es que antes de la extracción de la pieza, el uréter izquierdo es pasado por detrás del mesosigmoides laparoscópicamente.

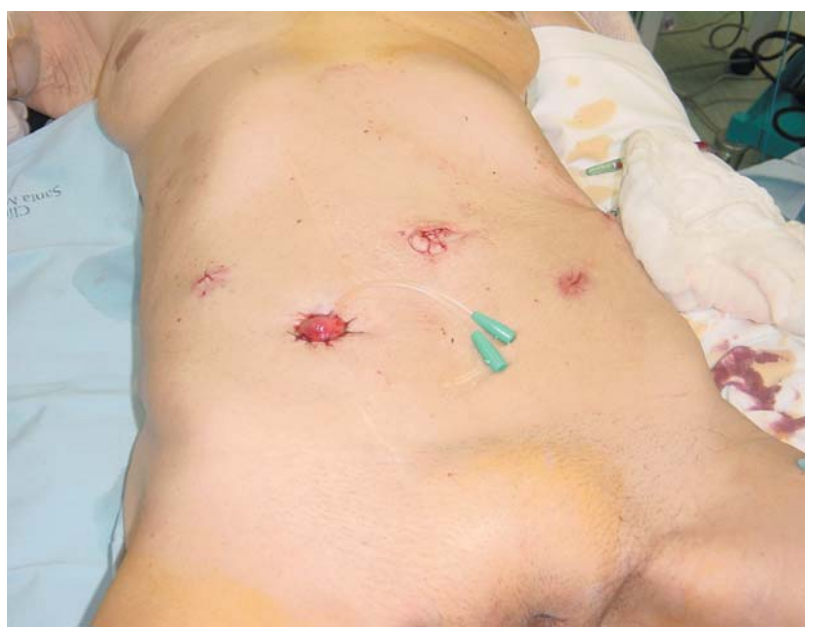

FIGURA 9

Reservorio continente tipo Indiana: Esta derivación la hemos efectuado en mujeres sin indicación oncológica de neovejiga ortotópica. No tenemos experiencia con la derivación tipo Mainz II, la cual podría ser una alternativa en estos casos. Usamos el segmento íleo-cecal, reconfigurándolo con sutura de Monocryl 3-0 y el segmento ileal, que constituirá la válvula se plica con una sutura a puntos separados de seda 2-0 o se adelgaza con varios disparos de TLC-55 sobre una sonda Nelaton 12 (Fig. 10). El estoma se ubica en el fondo del ombligo como un estoma plano, y se deja una sonda Nelaton $18 \mathrm{Fr}$ por el estoma y una sonda Pezzer 22 Fr como cistostomía por contrabertura (Fig. 11).

\section{TIPOS DE DERIVACIÓN URINARIA}

Se han publicado varios tipos de derivaciones urinarias en las series revisadas (Tabla 1). No existe una derivación ideal en cirugía abierta, tampoco existe esta en cirugía laparoscópica. El objetivo fundamental será realizar una derivación adecuada para cada paciente. La decisión no debe fundamentarse en las preferencias del cirujano por una 


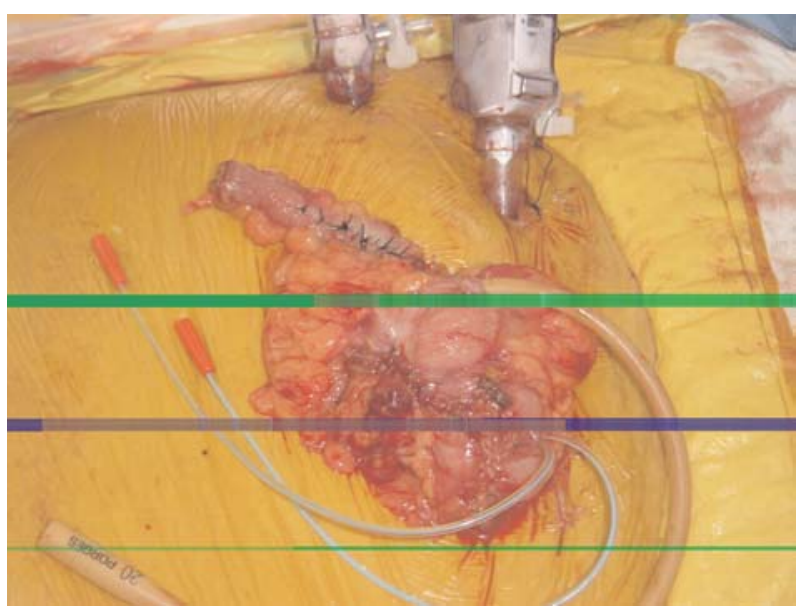

FIGURA 10

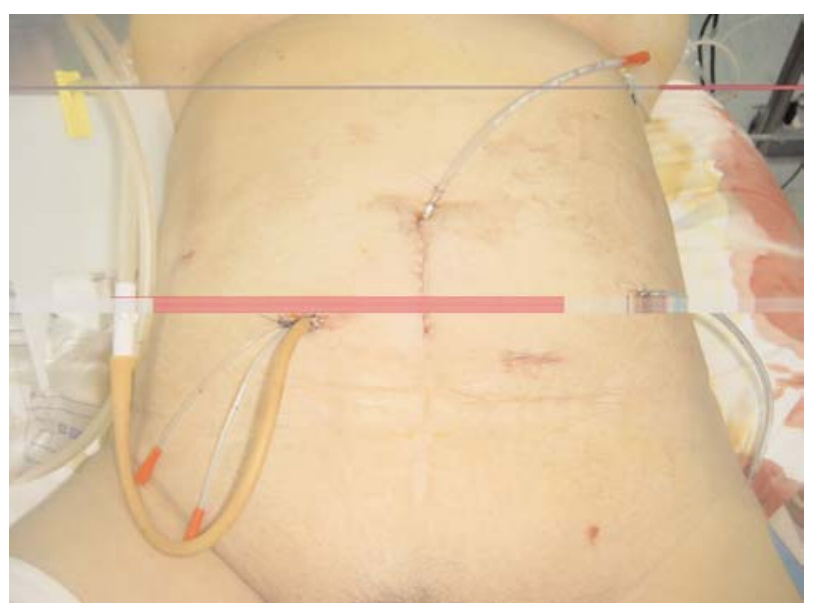

FIGURA 11

Tabla 1

\begin{tabular}{|c|c|c|c|c|c|c|c|c|c|c|}
\hline & $\mathbf{N}$ & Tipo derivación & $\begin{array}{l}\text { Tiempo } \\
\text { cirugia } \\
\text { (h) }\end{array}$ & $\begin{array}{l}\text { Sangrado } \\
\text { intraqx. } \\
\text { (cc) }\end{array}$ & $\begin{array}{l}\text { Estancia } \\
\text { hospital } \\
\text { (d) }\end{array}$ & $\begin{array}{l}\text { Complicaciones } \\
\text { mayores }\end{array}$ & Margen & $\begin{array}{c}\text { Linfa/ } \\
\text { no ganglios+ }^{\circ}\end{array}$ & $\begin{array}{l}\text { Seguimiento } \\
\text { o (meses) }\end{array}$ & $\begin{array}{l}\text { Supervivencia } \\
\text { № pacientes }\end{array}$ \\
\hline $\begin{array}{l}\text { Denewer } \\
1999^{42}\end{array}$ & 10 & CI minilap & 3.6 & - & $10-13$ & 5 pacientes? & NR & Obt/0 & - & 9 \\
\hline $\begin{array}{l}\text { Türk } \\
2001^{19}\end{array}$ & 5 & RS laparoscópico & $6.9-7.9$ & $190-300$ & 10 & No & - & Obt/0 & - & 5 \\
\hline $\begin{array}{l}\text { Gupta } \\
2002^{16}\end{array}$ & 5 & CI laparoscópico & $7-8$ & $300-400$ & $8-22$ & 1 obstrucción intestinal & - & $\mathrm{Obt} / 2$ & 24 & $\begin{array}{c}3 \text { (2 muertes causas } \\
\text { no relacionadas) }\end{array}$ \\
\hline $\begin{array}{l}\text { Gill } \\
2002^{18}\end{array}$ & 3 & $\begin{array}{l}2 \text { NOI laparoscópico } \\
1 \text { Indiana minilap }\end{array}$ & $8.5-10.5$ & $\begin{array}{c}300 \\
(200-400)\end{array}$ & $5-12$ & 1 hemorragia intestinal & - & Obt/0 & $5-19$ & 2 (1 muerte MTX) \\
\hline $\begin{array}{l}\text { Simonato } \\
2003^{7}\end{array}$ & 10 & $\begin{array}{c}6 \text { NOI minilap } \\
2 \text { US } \\
2 \text { cutáneas }\end{array}$ & $4.3-8.5$ & $\begin{array}{c}310 \\
(220-440)\end{array}$ & $5-9$ & $\begin{array}{c}2 \text { hidronefrosis } \\
\text { bilat, } 1 \text { hidronefrosis } \\
\text { unilateral }\end{array}$ & - & Obt/0 & $5-18$ & 10 (2 MTX) \\
\hline $\begin{array}{l}\text { Menon } \\
2003^{12}\end{array}$ & 17 & $\begin{array}{l}14 \text { NOI minilap } \\
3 \text { CI minilap }\end{array}$ & $4.3-5.4$ & $<150$ & NR & 1 sangrado postquirúrgico & - & Obt/1 & NR & 17 \\
\hline $\begin{array}{l}\text { Basillote } \\
2003^{11}\end{array}$ & 13 & NOI minilap & 8 & 1000 & 5.1 & 4 pacientes $^{2}$ & - & $\mathrm{Obt} / \mathrm{NR}$ & NR & 13 \\
\hline $\begin{array}{l}\text { Deger } \\
2004^{22}\end{array}$ & 20 & RS laparoscópico & $\begin{array}{c}8 \\
(6-13.8)\end{array}$ & $\begin{array}{c}200 \\
(190-800)\end{array}$ & $\begin{array}{c}15 \\
(11-30)\end{array}$ & $\begin{array}{l}2 \text { (1 fistula RS, } \\
1 \text { fistula } \\
\text { rectovaginal) }\end{array}$ & - & Ext/3 & $\begin{array}{c}33 \\
(13-42)\end{array}$ & $\begin{array}{l}18 \text { (3 MTX con } \\
2 \text { muertes) }\end{array}$ \\
\hline $\begin{array}{l}\text { Taylor } \\
2004^{24}\end{array}$ & 8 & IC minilap & 6.7 & $\begin{array}{c}637 \\
(400-1000)\end{array}$ & $\begin{array}{c}6.4 \\
(3-11)\end{array}$ & $\begin{array}{c}2 \text { (1 lesión rectal, } \\
1 \text { hemorragia intestinal) }\end{array}$ & 1 & Ext/1 & NR & NR \\
\hline
\end{tabular}

$\mathbf{N}$ : número de pacientes incluidos en la serie.

Tipo de derivación: tipo de derivación urinaria empleada y forma de realizarla.

CI: conducto ileal. NOI: neovejiga ortotópica ileal. Indiana: reservorio tipo Indiana pouch. RS: reservorio rectosigmoideo. US: ureterostomía sigmoidea.

Cutánea: ureterostomía cutánea bilateral. Minilap: creación de derivación intestinal a través de una pequeña incisión en la pared abdominal.

Laparoscópico: creación de la derivación intestinal de forma completamente laparoscópica.

Tiempo cirugia (h): tiempo quirúrgico medio en horas.

Sangrado intraqx (cc): sangrado reportado intraoperatorio en centímetros cúbicos.

Estancia hospitalaria (d): días de ingreso hospitalario.

Complicación: complicaciones postoperatorias en relación con la cirugía.

1. 1 lesión vascular, 1 hemorragia postoperatoria, 1 muerte perioperatoira, 1 fístula urinaria, 1 pielonefritis.

2. 1 obstrucción ureteral, 1 estenosis anastomosis neovejiga, 1 absceso epididimario, 1 dehiscencia de herida quirúrgica.

Margen: existencia de márgenes positivos.

Linfa/no ganglios: tipo de linfadenectomía realizada y número de pacientes con ganglios positivos.

Obt: linfadenectomía pélvica obturatríz. Ext: linfadenectomía pélvica extendida.

Seguimiento (meses): seguimiento publicado por las diferentes series expresado en meses.

Supervivencia $\mathbf{n}^{\circ}$ pacientes: número de supervivientes de la serie y causa de la muerte de los fallecidos.

MTX: enfermedad metastásica diseminada. 
determinada derivación, por su obligada realización laparoscópica o mediante cirugía convencional $^{2}$. Los modelos en animales de experimentación han contribuido de manera sustancial en la experiencia clínica ${ }^{8}$.

Para muchos autores la realización de la derivación es el verdadero handicap de la $\mathrm{CPRL}^{9,10}$. La dificultad de la sutura laparoscópica intracorpórea es el factor más importante que ha frenado el desarrollo de la laparoscopia urológica en el campo de la cirugía reconstructiva ${ }^{8}$.

\section{Técnicas de derivación}

La composición de la derivación urinaria se puede realizar de tres maneras diferentes. La alternativa más sencilla es realizarla de manera convencional, a través de una minilaparotomía ${ }^{1,11,12}$. Bastará una incisión de $7 \mathrm{~cm}$ en la pared abdominal para exteriorizar el fragmento intestinal y modelar el reservorio urinario ${ }^{8}$, su realización no parece reducir las ventajas de la cirugía laparoscópica ${ }^{9}$.

La segunda manera es exteriorizando las asas intestinales a través de los orificios de los trócares, confeccionando la derivación mediante sutura extracorpórea, ${ }^{4,13-15}$. En la realización de la sutura habrá que tener en cuenta la orientación de los tejidos, distorsionados por la tracción, y la extensa disección ureteral que se precisa para que los uréteres puedan llegar hasta la piel ${ }^{8,16}$.

Por último, se puede realizar mediante sutura intracorpórea laparoscópica ${ }^{16-22}$. La magnificación permite una excelente visión y una precisa aposición muco-mucosa de la anastomosis aunque, será imprescindible para su realización contar con una gran destreza en el manejo del instrumental laparoscópico 8,16 . Se cuestiona, según algunos autores, por prolongar innecesariamente el tiempo quirúrgico, ser técnicamente compleja y por desaprovechar la obligada minilaparotomía para la extracción de la pieza quirúrgica ${ }^{12}$. Esta opinión puede que cambie cuando contemos con medios de sutura automática laparoscópica más eficaces.

\section{LIMITACIONES DE LA CISTECTOMÍA RADICAL LAPAROSCÓPICA}

La CPRL se considera que todavía precisa de una mejor estandarización ${ }^{6,23}$. Con el tiempo, igual que ocurrió con la prostatectomía radical laparoscópica, se reducirán tiempos quirúrgicos y en un futuro no muy lejano no quedará limitada a centros de referencia ${ }^{6,7,9}$. Actualmente continúa siendo una cirugía costosa en términos económicos, que requiere de una larga curva de aprendizaje ${ }^{6}$.

Existen publicaciones que hablan a favor de iniciar la experiencia con la CPRL mediante la cirugía mano asistida ${ }^{13,24}$. De esta forma se mantienen las ventajas de la cirugía mínimamente invasiva, y se pretende acortar de esta manera la curva de aprendizaje $\mathrm{e}^{25}$.

\section{INDICACIONES}

Actualmente se seleccionan pacientes para la CPRL con neoplasias organoconfinadas no voluminosas. Debido al potencial aumento de la complejidad técnica, como contraindicaciones se citan la radioterapia pélvica previa, la quimioterapia neoadyuvante, la obesidad mórbida, pacientes con historia de múltiples cirugías abdominales, así como la infección activa intraabdominal y las diátesis hemorrágicas no corregidas $^{8,26}$.

\section{PRINCIPIOS QUIRÚRGICOS}

Principios oncológicos

La CRPL debe realizarse siguiendo los mismos principios de la cirugía oncológica convencional. Los indicadores de calidad en la CPRA están bien establecidos, relacionándose con la morbi-mortalidad, la afectación de los márgenes quirúrgicos, el número de ganglios linfáticos extraídos en la linfadenectomía y en número de procedimientos que debe realizar un centro para alcanzar estos estándares ${ }^{27}$.

Puppo et $\mathrm{al}^{6}$ revisaron $107 \mathrm{CPRL}$ publicadas, encontrando tres casos con márgenes positi$\operatorname{vos}^{11,24,28}$. Tres de las series publicadas mencionaban el número de ganglios linfáticos extraídos en la linfadenectomía ${ }^{16,28,29}$, variando el número entre 3 y 21 . Estos datos son similares a las largas series publicadas de CPRA ${ }^{30-32}$. La sorprendente baja tasa de márgenes positivos, incluso en estadios avanzados, se ha puesto en relación con la magnificación de imagen que ofrece la laparoscopia aunque también, y más probablemente, esta tenga más que ver con la selección de los pacientes intervenidos mediante esta técnica ${ }^{6}$. 


\section{Control oncológico}

Es muy importante en cirugía laparoscópica ser cuidadoso con la manipulación del carcinoma de células transicionales para evitar su diseminación local ${ }^{18}$. Se ha comunicado el seguimiento de los pacientes en aproximadamente el 50\% de los casos publicados, con un tiempo máximo de seguimiento de 48 meses y un seguimiento medio de 11,5 meses ${ }^{6}$. El corto seguimiento disponible hasta el momento y el número limitado de casos publicados, no permite extraer conclusiones definitivas sobre si la CPRL es una cirugía oncologicamente segura a largo plazo. En un primer análisis, no parece que la CPRL tenga un impacto negativo en la supervivencia de los pacientes ${ }^{6}$.

\section{Calidad de vida}

La calidad de vida de los pacientes oncológicos es uno de los retos más importantes de la medicina del futuro. La preservación de la continencia y la función sexual es muy importante, especialmente en los pacientes jóvenes sometidos a una cistectomía radical $^{33}$. Las series de cistectomía radical con preservación de los haces neurovasculares consiguen mantener la potencia sexual entre un 40 y un $60 \%$ de los pacientes ${ }^{33}$. La preservación de la próstata en la cistectomía radical con derivación urinaria ortotópica, tanto en cirugía abierta ${ }^{34}$ como en laparoscópica ${ }^{35}$, ofrece prometedores resultados respecto a la continencia y conservación de la potencia sexual. No obstante la falta de estudios a largo plazo obliga a ser cautos en sus indicaciones ${ }^{33}$.

\section{CISTECTOMÍA RADICAL: LAPAROSCÓPICA VS. ABIERTA}

Ventajas

La reducción en unos pocos centímetros de la longitud de la incisión quirúrgica, no es suficiente para justificar un cambio tan radical en la cirugía urológica ${ }^{6}$.

Las potenciales ventajas de la CPRL hay que buscarlas en la reducción del sangrado intraoperatorio, una mejor visualización de las estructuras, la deambulación más precoz en el postoperatorio inmediato y en la reducción de la estancia hospitalaria, las necesidades de analgesia y el íleo adinámico tras la cirugía ${ }^{2}$.

La reducción del sangrado intraoperatorio a $300-400 \mathrm{ml}^{26}$, parece relacionada con la magnifi- cación que ofrece la laparoscopia, ya que permite una disección y hemostasia más cuidadosa. La presión intra-abdominal del pneumoperitoneo también contribuye a reducir el sangrado ${ }^{26}$. Basillote et $\mathrm{al}^{11}$ no encontraron diferencias en relación al sangrado en su serie comparativa entre CPRL y CPRA, tal vez porque fue realizada cuando todavía estaban iniciando su experiencia en laparoscopia. Las series laparoscópicas publicadas por Gill et $\mathrm{al}^{18} \mathrm{y}$ Türk et $\mathrm{al}^{19}$ presentan sangrados significativamente menores a la cirugía abierta ${ }^{36}$.

La reducción de la estancia hospitalaria no parece ser muy diferente entre la CPRL y la $\mathrm{CPRA}^{6}$, a pesar de que algunos autores ${ }^{11}$ encuentren diferencias significativas en sus series. Esto se explica porque el alta se relaciona con la recuperación del tránsito intestinal y con la posibilidad de que el paciente tenga apoyo fuera del hospital para continuar su recuperación ${ }^{6}$. Por el contrario, si parece que con la CPRL el paciente pueda retornar a su actividad diaria de forma más precoz ${ }^{26}$.

Las necesidades de analgesia en el postoperatorio si son significativamente menores en la CPRL. Esto contribuye, junto con la menor manipulación de los intestinos durante la cirugía, a la menor incidencia de íleo adinámico en el postoperatorio $^{11,26,37,38}$.

Ventajas más difíciles de medir de la laparoscopia son la menor respuesta inflamatoria y la menor inmunosupresión por la agresión quirúrgica según destacan investigaciones recientes ${ }^{39,40}$. Como consecuencia sería esperable una menor tasa de infecciones, íleo adinámico y una reducción en la respuesta inflamatoria no específica.

\section{Inconvenientes}

Existen una serie de desventajas a la hora de realizar la CPRL. La prolongación del tiempo quirúrgico, es un problema que parece solucionado, puesto que se ha reducido desde las primeras series publicadas ${ }^{18-20}$ de entre 7 y 11 horas a entre 4 y $8^{38}$. Actualmente, los centros con experiencia, realizan la CPRL en tiempos competitivos respecto a la $\mathrm{CPRA}^{7,11,13,19,24}$. Otro importante handicap de la CPRL es su curva de aprendizaje ${ }^{6}$, que resulta especialmente dificultosa, sobre todo 
para cirujanos sin experiencia previa en cirugía radical prostática laparoscópica.

El coste económico es otro obstáculo para cualquier cirugía laparoscópica ${ }^{6}$. Precisa de instrumentos de alta tecnología, lo que incrementa los costes económicos directos.

Cistoprostatectomía radical robótica:

La utilización de dispositivos robóticos puede compensar la falta de percepción táctil de la cirugía laparoscópica, reduciendo la curva de aprendizaje $^{26}$. Esto lo consigue mediante la mejora en la percepción espacial tridimensional, la magnificación visual de hasta diez veces respecto a la realidad y la mayor libertad de movimientos con los instrumentos laparoscópicos ${ }^{41}$. Existen en la literatura trabajos prometedores en cuanto a la utilidad y resultados obtenidos con la robótica en la CPRL $^{12,17}$.

\section{MORBILIDAD Y COMPLICACIONES}

\section{Mortalidad}

La mortalidad comunicada de las grandes series de CPRA es inferior al 4\% $\%^{31-33}$. En relación con 107 casos revisados, Puppo et al ${ }^{6}$ sólo encontraron un caso de muerte en relación con la cirugía ${ }^{42}$.

\section{Complicaciones}

Se ha comunicado un caso de lesión rectal en un paciente que previamente había recibido radioterapia pélvica ${ }^{24}$. En la serie inicial de 21 CPRL en la Cleveland Clinic, se produjeron 6 complicaciones mayores (29\%) y 9 menores (45\%). Todas las complicaciones mayores precisaron reintervención quirúrgica (3 obstrucciones intestinales, 1 fístula de la anastomosis ureteroileal, 1 fístula uretro-vaginal y 1 perforación intestinal). Las complicaciones menores fueron esencialmente relacionadas con un íleo prolonga$\mathrm{do}^{26}$. Hemal $^{43}$ comunica en su serie de $11 \mathrm{CPRL}$ 5 complicaciones. Tres intraoperatorias (1 lesión de la vena iliaca externa, 2 pequeñas lesiones rectales) y 2 relacionadas con la cirugía laparoscópica (enfisema subcutáneo e hipercapnia).

Puppo et $\mathrm{al}^{6}$, revisaron 107 casos publicados de CPRL, encontrando 27 complicaciones en relación con la cirugía. Esta cifra es similar al 25\% de morbilidad que aparece en las grandes series de CPRA.

\section{CONCLUSIONES}

La cistectomía radical laparoscópica es un procedimiento que demanda entrenamiento extensivo en cirugía reconstructiva laparoscópica. El tiempo quirúrgico, especialmente al inicio de la experiencia, es mayor que el de la cirugía abierta. Sin embargo, nuestra experiencia inicial demuestra que cumple los estándares oncológicos de la cirugía del cáncer vesical.

Tiene ventajas potenciales sobre la cistectomía abierta, como son menor sangrado, menor necesidad de analgesia, deambulación precoz, menor íleo postoperatorio, menor convalecencia. Estas ventajas deberán ser evaluadas sobre la base de experiencias mayores, en centros de referencia, antes de pasar a constituir un procedimiento de real alternativa frente a la cirugía tradicional.

\section{REFERENCIAS}

1. Sorcini A, Tuerk I. Laparoscopic radical cystectomy with ileal conduit urinary diversion Urol Oncol. 2004;22(2): 149-152.

2. Canes D, Triaca V, Tuerk I. Laparoscopic radical cystectomy with continent urinary diversion. Curr Urol Rep. 2005; 6(2): 109-117.

3. Dalbagni G, Genega E, Hashibe M, Zhang ZF, Russo P, Herr H, Reuter V. Cystectomy for bladder cancer: a contemporary series J Urol 2001;165(4):1111-1116.

4. Sánchez de Badajoz E, Gallego Perales JL, Reche Rosado A, Gutiérrez de la Cruz JM, Jiménez Garrido A.. Laparoscopic cystectomy and ileal conduit: a case report $\mathrm{J}$ Endourol. 1995;9(1):59-62.

5. Puppo P, Perachino M, Ricciotti G, Bozzo W, Gallucci M, Carmignani G. Laparoscopically assisted transvaginal radical cystectomy. Eur Urol 1995;27(1):80-84.

6. Puppo P, Naselli A. Laparoscopic radical cystectomy. Curr Urol Rep 2005;6(2):106-108.

7. Simonato A, Gregori A, Lissiani A, Bozzola A, Galli S, Gaboardi F. Laparoscopic radical cystoprostatectomy: a technique illustrated step by step Eur Urol. 2003;44(1): 132-138.

8. Fergany AF, Novick AC, Gill IS. Laparoscopic urinary diversion. World J Urol. 2000;18(5):345-348.

9. Velthoven RF, Piechaud T. Laparoscopic radical cystectomy with ileal conduit diversion. Curr Urol Rep. 2005; 6(2): 93-100.

10. Andersen KR, Clayman RU. Laparoscopic lower urinary tract reconstruction World J Urol. 2000;18(5):349-354.

11. Basillote JB, Abdelshehid C, Ahlering TE, Shanberg AM. Laparoscopic assisted radical cystectomy with ileal neobladder: a comparison with the open approach. J Urol. 2004;172(2):489-493.

12. Menon M, Hemal AK, Tewari A, Shrivastava A, Shoma AM, El-Tabey NA, Shaaban A, Abol-Enein H, Ghoneim MA. Nerve-sparing robot-assisted radical cystoprostatectomy and urinary diversion BJU Int. 2003;92(3):232-236. 
13. Peterson AC, Lance RS, Ahuja SK. Laparoscopic hand assisted radical cystectomy with ileal conduit urinary diversionJ Urol. 2002;168(5):2103-2105.

14. Kozminski M, Partmian KO. Case report of laparoscopic ileal loop conduit J Endourol. 1992;6:147-150.

15. Vara-Thorbeck C, Sanchez de Badajoz E. Laparoscopic ileal-loop conduit Surg Endosurg. 1994;8(2):114-115.

16. Gupta NP, Gill IS, Fergany A, Nabi G. Laparoscopic radical cystectomy with intracorporeal ileal conduit diversion: five cases with 2-year follow up BJU Int. 2002;90(4):391-396.

17. Beecken WD, Wolfram M, Engl T, Bentas W, Probst M, Blaheta R, Oertl A, Jonas D, Binder J. Robotic-assisted laparoscopic radical cystectomy and intra-abdominal formation of an orthotopic ileal neobladder Eur Urol. 2003; 44(3):337-349.

18. Gill IS, Kaouk JH, Meraney AM, Desai MM, Ulchaker JC, Klein EA, Savage SJ, Sung GT. Laparoscopic radical cystectomy and continent orthotopic ileal neobladder performed completely intracorporeally: the initial experience $\mathrm{J}$ Urol. 2002;168(1):13-18.

19. Turk I, Deger S, Winkelmann B, Schonberger B, Loening SA.. Laparoscopic radical cystectomy with continent urinary diversion (rectal sigmoid pouch) performed completely intracorporeally: the initial 5 cases $\mathrm{J}$ Urol. 2001;165 (6): 1863-1866.

20. Gill IS, Fergany A, Klein EA, Kaouk JH, Sung GT, Meraney AM, et al. Laparoscopic radical cystectoprostatectomy with ileal conduit performed completely intracorporeally: the initial 2 cases Urology. 2000;56(1):26-30.

21. Potter SR, Charambura TC, Adams JB 2nd, Kavoussi LR. Laparoscopic ileal conduit: five-year follow-up Urology. 2000;56(1):22-25.

22. DeGer S, Peters R, Roigas J, Wille AH, Tuerk IA, Loening SA. Laparoscopic radical cystectomy with continent urinary diversion (rectosigmoid pouch) performed completely intracorporeally: an intermediate functional and oncological analysis Urology. 2004;64(5):935-939.

23. Hrouda D, Adeyoju A AB, Gill IS. Laparoscopic radical cystectomy and urinary diversion: fad or future. BJU Int. 2004;94(4):501-505.

24. Taylor GD, Duchene DA, Koeneman KS. Hand assisted laparoscopic cystectomy with minilaparotomy ileal conduit: series report and comparison with open cystectomy $\mathrm{J}$ Urol. 2004;172(4 Pt 1):1291-1296.

25. Wolf JS, Moon TD, Nakade SY. Hand assisted laparoscopic nephrectomy: comparison to standard laparoscopic nephrectomy J Urol. 1998;160(1):22-27.

26. Ukimura O, Moinzadeh A, Gill IS. Laparoscopic radical cystectomy and urinary diversion Curr Urol Rep. 2005;6 (2):118-121.

27. Herr H, Lee C, Chang S, Lerner S Standardization of radical cystectomy and pelvic lymph node dissection for bladder cancer: a collaborative group report J Urol. 2004;171 (5): $1823-1828$.

28. Hemal AK, Singh I, Kumar R. Laparoscopic radical cystectomy and ileal conduit reconstruction: preliminary experience. J Endourol. 2003;17(10):911-916.

29. Menon M, Hemal AK, Tewari A, Shrivastava A, Shoma AM, Abol-Ein H, Ghoneim MA. Robot-assisted radical cystectomy and urinary diversion in female patients: technique with preservation of the uterus and vagina $\mathrm{J}$ Am Coll Surg. 2004;198(3):386-393.
30. EORTC-GU Group. Neoadjuvant cisplatin, methotrexate, and vinblastine chemotherapy for muscle-invasive bladder cancer: a randomized, controlled trial. International collaboration of trialists. Lancet. 1999;354(9178):533-540.

31. Ghoneim MA, El-Mekresh MM, El-Baz MA, El-Attar IA, Ashamallah A. Radical cystectomy for carcinoma of the bladder: critical evaluation of the results in 1026 cases $\mathrm{J}$ Urol. 1997;158(2):393-399.

32. Stein JP, Lieskovsky G, Cote R, Groshen S, Feng AC, Boyd $\mathrm{S}$, et al. Radical cystectomy in the treatment of invasive bladder cancer: long-term results in 1054 patients. J Clin Oncol. 2001;19(3):666-675.

33. Cestari A, Naspro R, Riva M, Bellinzoni P, Nava L, Rigatti P, Guazzoni G. Nerve-sparing laparoscopic cystectomy Curr Urol Rep. 2005;6(2):101-105.

34. Colombo R, Bertini R, Salonia A, Da Pozzo LF, Montorsi F, Brausi M, Roscigno M, Rigatti P. Nerve and seminal sparing radical cystectomy with orthotopic urinary diversión for select patients with superficial bladder cancer: an innovative surgical approach J Urol. 2001;165(1):51-55.

35. Guazzoni G, Cestari A, Colombo R, Lazzeri M, Montorsi F, Nava L, Losa A, Rigatti P. Laparoscopic nerve and seminalsparing cystectomy with orthotopic ileal neobladder: the first three cases Eur Urol. 2003;44(5):567-572.

36. Chang SS, Smith JA, Cookson MS. Decreasing blood loss in patients treated with radical cystectomy: a prospective, randomized trial using a new stapling device $\mathrm{J}$ Urol. 2003;169(3):951-954.

37. Gaboardi F, Simonato A, Galli S, Lissiani A, Gregori A, Bozzola A. Minimally invasive laparoscopic neobladder J Urol. 2002;168(3):1080-1083.

38. Maxwell-Armstrong CA, Robinson MH, Scholefield JH. Laparoscopic colorectal cancer surgery Am J Surg. 2000; 179(6):500-507.

39. Targarona EM, Balague C, Knook MM, Trias M. Laparoscopic surgery and surgical infection Br J Surg. 2000;87(5): 536-544.

40. Busby E, Das S, Rao Tunuguntla HS, Evans CP. Handassisted laparoscopic vs the open (flank incisión) approach to radical nephrectomy BJU Int. 2003;91(4):341-344.

41. Shah NL, Hemal AK, Menon M. Robot-assisted radical cystectomy and urinary diversion Curr Urol Rep. 2005;6(2): 122-125.

42. Denewer A, Kotb S, Hussein O, El-Maadawy M. Laparoscopic assisted cystectomy and lymphadenectomy for bladder cancer: initial experience World J Surg. 1999;23 (6):608-611.

43. Hemal AK, Kumar R, Seth A, Gupta NP. Complications of laparoscopic radical cystectomy during the initial experience Int J Urol. 2004;11(7):483-488.

44. Abdel-Hakim AM, Bassiouny F, Abdel Azim MS, Rady I, Mohey T, Habib I, Fathi H.. Laparoscopic radical cystectomy with orthotopic neobladder J Endourol. 2002;16(6): 377-381.

Dr. Octavio Castillo

E-mail: octaviocastillo@vtr.net 